\title{
Erratum: Synergistic impeding of phonon transport through resonances and screw dislocations [Phys. Rev. B 103, 085414 (2021)]
}

\author{
Hongying Wang, Yajuan Cheng, Masahiro Nomura, Sebastian Volz, Davide Donadio, \\ Xiaohong Zhang, and Shiyun Xiong \\ (Q) (Received 16 August 2021; published 3 September 2021)
}

DOI: 10.1103/PhysRevB.104.119903

Another strategy of enhancing phonon scattering was reported earlier in SiGe nanowires by Al-Ghalith et al. [1] where screw dislocation and surface ripples were combined together.

[1] J. Al-Ghalith, Y. Ni, and T. Dumitrica, Nanowires with dislocations for ultralow lattice thermal conductivity, Phys. Chem. Chem. Phys. 18, 9888 (2016). 